\title{
Turkish League Against Rheumatism Consensus Report: Recommendations For Management of Axial Spondyloarthritis
}

Hatice BODUR ${ }^{1}$, Fatma Gül YURDAKUL ${ }^{2}$, Şebnem ATAMAN³ , Yeşim GARİ² ${ }^{2}$, Kemal NAS ${ }^{4}$, Fikriye Figen AYHAN ${ }^{5,6}$, Özgür AKGÜL Ayşen AKINCI ${ }^{8}$, Zuhal ALTAY ${ }^{9}$, Murat BİRTANE ${ }^{10}$, Derya SOY BUĞDAYCI ${ }^{11}$, Erhan ÇAPKIN ${ }^{12}$, Remzi ÇEVİK ${ }^{13}$, Tuncay DURUÖZ ${ }^{14}$,

Gülcan GÜRER ${ }^{15}$, Cahit KAÇAR ${ }^{16}$, Ayhan KAMANLI ${ }^{17}$, Ece KAPTANOĞLU ${ }^{18}$, Taciser KAYA ${ }^{19}$, Hilal KOCABA\$ุ\$ ${ }^{20}$, Ömer KURU ${ }^{21}$, Meltem Alkan MELİKOĞLU ${ }^{22}$, Erhan ÖZDEMİREL ${ }^{23}$, Sumru ÖZEL ${ }^{24}$, Aylin REZVANİ² ${ }^{25}$ İlhan SEZER ${ }^{26}$, İsmihan SUNAR ${ }^{3}$, Gürkan YILMAZ ${ }^{3}$

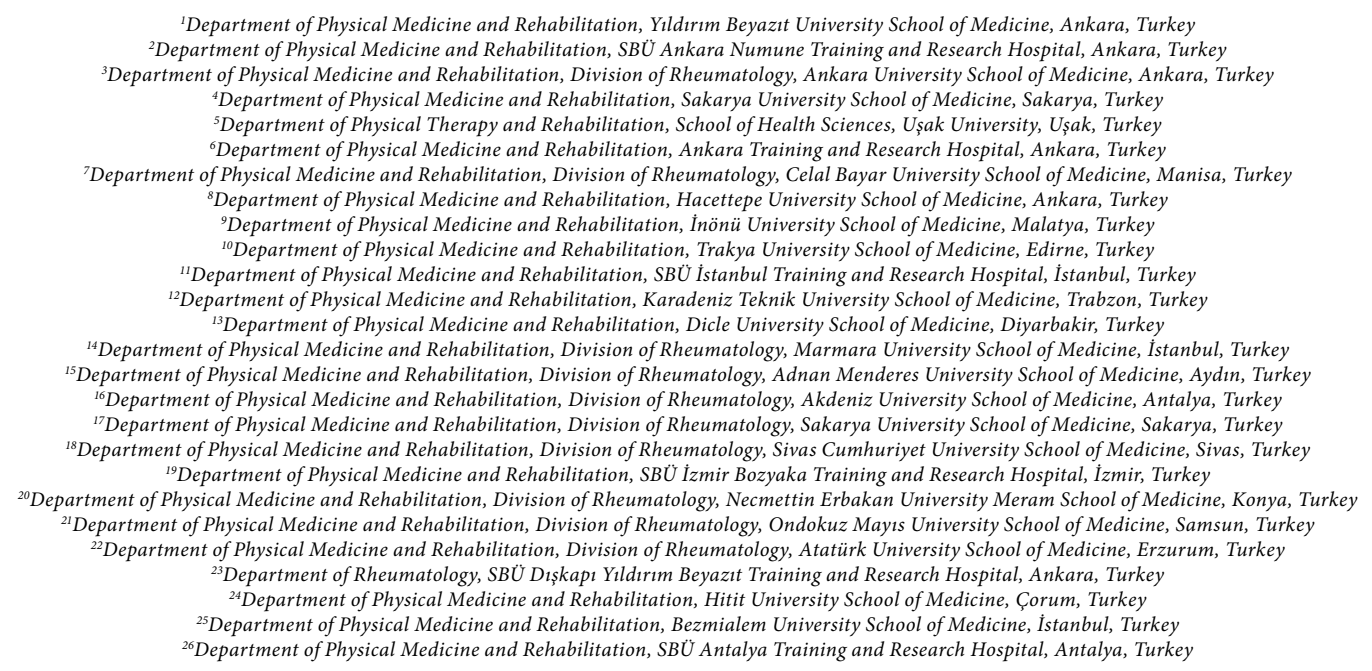

\section{ABSTRACT}

Objectives: This study aims to update 2011 Turkish League Against Rheumatism SpondyloArthritis Recommendations, and to compose a national expert opinion on management of axial spondyloarthritis under guidance of current guidelines, and implantation and dissemination of these international guidelines into our clinical practice.

Materials and methods: A scientific committee of 28 experts consisting of 14 rheumatologists and 14 physical medicine and rehabilitation specialists (one of them also has an immunology PhD) was formed. The recommendations, systematic reviews, and meta-analyses including pharmacologic and non-pharmacologic treatment were scrutinized paying special attention with convenient key words. The draft of Turkish League Against Rheumatism opinion whose roof consisted of international treatment recommendations, particularly the Assessment of SpondyloArthritis International Society/European League Against Rheumatism recommendations was composed. Assessment of level of agreement with opinions by task force members was established through the Delphi technique. Voting using a numerical rating scale assessed the strength of each recommendation.

Results: Panel compromised on five basic principles and 13 recommendations including pharmacological and nonpharmacological methods. All of the recommendations had adequate strength.

Conclusion: Turkish League Against Rheumatism expert opinion for the management of axial spondyloArthritis was developed based on scientific evidence. These recommendations will be updated regularly in accordance with current developments.

Keywords: Axial spondyloarthritis; comorbidities; exercises; nonpharmacological management; treatment.

Received: January 12, 2018 Accepted: February 10, 2018 Published online: February 14, 2018

Correspondence: Hatice Bodur, MD. Yıldırım Beyazıt Üniversitesi Tıp Fakültesi Fiziksel Tıp ve Rehabilitasyon Anabilim Dalı, 06010 Ankara, Turkey.

Tel: +90 312 - 5084810 e-mail: haticebodur@gmail.com

@2018 Turkish League Against Rheumatism. All rights reserved. 
The term 'spondyloArthritis' (SpA) describes a group of diseases with distinct clinical manifestations. Axial spondyloarthritis (ax-SpA) is a chronic inflammatory disease affecting the axial skeleton (spinal and sacroiliac joints) predominantly. Human leukocyte antigen B-27 positivity is common among these patients. Peripheral arthritis, enthesitis, dactylitis as well as extraarticular manifestations of $\mathrm{spA}$ including psoriasis, anterior uveitis, and inflammatory bowel diseases may be present. ${ }^{1}$

In current medical terminology, inflammatory disease of the spine is named as SpA. This term brings the question whether ax-SpA and ankylosing spondylitis (AS) refer to same disease. Sacroiliitis can be detected at early non-radiographic phase by magnetic resonance imaging (MRI). Some of these patients develop radiographic AS and structural destruction while others remain as non-radiographic ax-SpA. In other words, AS and non-radiographic ax-SpA represent the different phases of the same clinical entity. Due to the development of efficient novel therapeutics, early diagnosis is of great significance. ${ }^{2}$

Physicians pursue current guidelines or recommendations in order to stay informed of evidence-based data. While clinical trials provide complicated information, guidelines offer special statements out of these data. In this way, guidelines may serve physicians in their clinical practices. ${ }^{3}$

In recent years, several guidelines regarding management of SpA including the Assessment of SpondyloArthritis International Society (ASAS) and European League Against Rheumatism (EULAR) Recommendations have been published parallel with the novelties in early diagnosis and treatment. ${ }^{4-17}$ Turkish League Against Rheumatism (TLAR) published the recommendations on management of SpA in 2011 for the first time. ${ }^{18}$ In this study, we aimed to update 2011 TLAR SpA Recommendations, and to compose a national expert opinion on management of ax-SpA under guidance of current guidelines, and implantation and dissemination of these international guidelines into our clinical practice.

\section{METHODOLOGY}

The first meeting was held on June 2017. The aim of the project was to make a consensus on TLAR opinions including the updates in management of ax-SpA. The target population was physicians from specialties dealing with SpA patients, particularly rheumatology and physical medicine and rehabilitation specialists. A conveyor (project director) and a fellow were employed for the literature search.

\section{COLLECTION OF EVIDENCE}

The systematic literature search included articles in English published in 2012-2017 in PubMedMedline, and Scopus. The recommendations, systematic reviews, and meta-analyses including pharmacologic and non-pharmacologic treatment were scrutinized paying special attention with convenient key words. Of the studied articles, 14 were guidelines. ${ }^{4-17}$ The articles were analyzed, summarized, and discussed. The draft of TLAR opinion whose roof consisted of international treatment recommendations, particularly the ASAS/EULAR Recommendations was composed. As well as containing certain original concepts, general structure of the draft pursued model of other international recommendations.

Experts decided to commence with five basic principles considered to be exact and comprehensive in current treatment and management of Ax-SpA. In 2011 TLAR Ankylosing Spondylitis National Treatment Recommendations, basic principles and recommendations were not handled separately; however, on noticing that overarching principles and recommendations were two different headlines in current guidelines, we considered this approach would be more beneficial in clinical practice.

This draft was discussed in another meeting with other project coordinators. Following this meeting, the final form of the draft was sent to expert panel members consisting of 14 rheumatologists and 14 physical medicine and rehabilitation specialists (one of them also has an immunology $\mathrm{PhD}$ ) to appeal their remarks. Their opinions and contributions were taken through e-mail. Assessment of level of agreement with opinions by task force members was established through the Delphi technique. In numerical scale; 0 meant 'I fully disagree', and 10 meant 'I fully 


\begin{tabular}{ll} 
Table 1. Strength of recommendation \\
\hline Strength \\
\hline A & $\begin{array}{l}\text { Category I evidence } \\
\text { B }\end{array}$ \\
C & $\begin{array}{l}\text { Category II evidence or extrapolated from category I } \\
\text { Category III evidence or extrapolated from category I } \\
\text { or II evidence }\end{array}$ \\
D & $\begin{array}{l}\text { Category IV evidence or extrapolated from category II } \\
\text { or III evidence }\end{array}$
\end{tabular}

agree'. In the first round, level of agreement (LoA) was eight or above for each item. In the second meeting held on October 2017, contributions and remarks of the experts were brought forward for discussion and suggestions by the conveyor without announcing the identity of the contributors. The second Delphi round for the final form of recommendations was performed with keypads.

Level of agreement and strength of recommendation (SOR) were evaluated by Numerical Scale. SORs (Table 1 and Table 2) were categorized using the methodology according to 2014 update of the EULAR standardized operating procedures. ${ }^{3}$

\section{Basic Principle 1}

Axial spondyloarthritis is a potentially severe disease. A multidisciplinary approach in coordination of a rheumatologist or physical medicine and rehabilitation specialist is required for the management of musculoskeletal and extraarticular manifestations (LoA=9.29 \pm 2.22).

As depicted before in the ASAS/EULAR Recommendations, National Institute for Health and Care Excellence (NICE) guidelines, and prior TLAR Recommendations, this item points out that ax-SpA is a severe disease with several extraarticular manifestations and comorbid situations. , $, 6,18^{2}$

The standardized morbidity rates are increased for uveitis, inflammatory bowel disease, psoriasis, osteoporosis and atrioventricular blocks. Ischemic heart disease and hypertension are other comorbid situations with increased frequency. ${ }^{19,20}$ It was emphasized that the treatment in SpA should be adjusted considering the extraarticular symptoms and signs, and comorbidities and that the most convenient treatment may be achieved when
Table 2. Category of evidence

\begin{tabular}{|c|c|}
\hline Ia & Meta-analysis of randomized controlled trials \\
\hline $\mathrm{Ib}$ & Randomized controlled trial \\
\hline IIa & Controlled study without randomization \\
\hline $\mathrm{IIb}$ & Quasi-experimental study \\
\hline III & $\begin{array}{l}\text { Non-experimental descriptive studies, such as comparative, } \\
\text { correlation and case-control studies }\end{array}$ \\
\hline IV & $\begin{array}{l}\text { Expert committee reports or opinion or clinical experience } \\
\text { of respected authorities, or both }\end{array}$ \\
\hline
\end{tabular}

collaborated with relevant specialists in case of multisystem involvement. ${ }^{5,18,19}$ Rheumatology or physical medicine and rehabilitation specialists should be the coordinator in this multidisciplinary approach.

Rheumatology is a relatively new field of science in Turkey and in the world. In our country, it is a subspecialty after completing the residency in either physical medicine and rehabilitation or internal medicine. Since the number of rheumatologists is inadequate in our country, physical medicine and rehabilitation specialists are intensely interested in the diagnosis, treatment, and rehabilitation of rheumatic diseases in addition to musculoskeletal and neurological diseases. The primary reason for this application is that such training and skills occupy a highly important place in the main curriculum of physical medicine and rehabilitation residency. ${ }^{21}$ As seen in composition of the expert panel, the responsible physicians of this disease in our country are rheumatologists and physical medicine and rehabilitation specialists.

\section{Basic Principle 2}

The main aims of the treatment of patients with ax-SpA are to control the inflammation and symptoms, to prevent structural damage, to minimize comorbidities, to avoid adverse effects of the treatment, normalization and preservation of functionality and social participation, and maximization of the health-related quality of life (LoA=9.89 \pm 0.31$)$.

Previously in 2011 TLAR Recommendations, the treatment target was not mentioned with a certain item. Considering the significance of treat to target strategy, the necessity of referring to aim of the treatment has emerged. The possibility of early diagnosis and availability of novel therapeutic options enable treat to target strategy of rheumatoid arthritis (RA) also applicable in 
SpA. The ideal target should regard clinical signs, objective indicators of the inflammation, structural damage, physical function, and quality of life..$^{22}$ In the 2017 update of recommendations by International Task Force, aim of the treatment is mentioned within the first basic item in overarching principles. ${ }^{5}$

Comorbid situations are of great significance since they may affect survival and quality of life more than the primary disease does, and limit therapeutic options and treatment success. In this item, minimizing the comorbid conditions was emphasized. ${ }^{5,19}$ Main comorbid diseases are osteoporosis, uveitis (anterior), aortitis, aortic insufficiency, psoriasis, inflammatory bowel disease, spinal injuries, cauda equina syndrome, erectile dysfunction, restrictive lung disease, ischemic heart disease, stroke, joint replacement, hyperlipidemia/metabolic syndrome, surgery, major depression, and alcoholism. ${ }^{6}$ Efficient treatment of the primary disease and comorbidities normalizes functional status and social participation, and increases the quality of life.

\section{Basic Principle 3}

\section{Non-pharmacological}

and pharmacological treatment methods should be combined in the ideal treatment of the patients with ax-SpA (LoA=9.25 \pm 2.35$)$.

This principle is exactly the same as the fourth item of TLAR Recommendations. ${ }^{18}$ The treatment goal of ax-SpA is suppressing the inflammation, and providing and maintaining the maximum functional status and quality of life. This is solely possible by combining the pharmacological and non-pharmacological (education, exercise, physical medicine and rehabilitation) treatment methods. ${ }^{15}$ While exercise constitutes the most important part of non-pharmacological treatment, it also includes maximizing the patient education, motivation for better compliance, and smoking cessation. ${ }^{23}$

Assessment of SpondyloArthritis International Society/EULAR also added this item to basic principles depending on significance of non-pharmacological approaches in $\mathrm{Ax}-\mathrm{SpA}$ compared to other inflammatory arthritides. ${ }^{4}$ The expert panel discussed that non-pharmacological methods were often omitted in clinical practice and that it should be handled meticulously.

\section{Basic Principle 4}

The treatment of ax-SpA should rely on the shared decision between patient and physician, and should aim the best treatment and care $(L o A=9.36 \pm 1.74)$.

In the fourth item of 2011 TLAR Recommendations; it was underlined that request and expectations of patients should be taken into account. ${ }^{18}$ The shared decision between the patient and physician is important as is in other diseases and discussed in a more detailed way than prior recommendations. The shared decision involves all treatment stages. This necessitates sufficient knowledge about the disease and informing the patient properly regarding risk and benefits of different therapeutic options. This approach would also enhance treatment compliance and success. ${ }^{14}$ Treatment choice should be made discussing the advantages and disadvantages of current medications between the clinician and the patient. This may also include related situations such as extra-articular manifestations. ${ }^{6}$

The expert panel decided that 'the best care' that is noted as an important issue in ASAS/ EULAR Update Recommendations should be a part of the basic principles. ${ }^{4}$ The physician and patient together make a decision for the best treatment and care considering all risks, benefits, requirements, and expectations. In other words, the best care is tailor-made for each patient and it should be endeavored to attain the target. ${ }^{4,5}$

\section{Basic Principle 5}

Axial spondyloarthritis has high personal, medical, and societal costs. All of them should be kept in mind in the management of the disease $(\mathrm{LoA}=9.18 \pm 1.76)$.

An item regarding cost of the treatment was not available in 2011 TLAR Recommendations. The cost of the treatment has recently drawn attention while it was not underscored previously. Ax-SpA is a disease with emerging novel therapeutic options among which considerable cost differences are available. This item has arisen due to targeted treatment, individualized treatment, and high 
medical treatment costs. ${ }^{4}$ Considering pharmacoeconomical data, determining the disease burden, and analysis of direct and indirect costs were dealt in general treatment principles and ethical principles of 2014 update on the Canadian Rheumatology Association/the Spondyloarthritis Research Consortium of Canada Treatment Recommendations for the Management of SpA for the first time..$^{10}$ Also, 2017 NICE Guideline refers to the significance of economical analysis in treatment choice. ${ }^{6}$

When a treatment choice will be made, best treatment and care principle should be considered. However, if the same outcome is anticipated for each therapeutic option, medical costs may affect the choice of treatment. The term 'individual' was included in the item to emphasize that 'the best care' is important and changes from a patient to another. ${ }^{4,6}$

\section{Recommendation 1}

The treatment of ax-SpA patients should be individualized considering current symptoms and signs of the disease (axial, peripheral, extra-articular), comorbid situations, psychosocial factors, requests, and expectations of the patients $($ LoA $=9.36 \pm 1.74)$.

The treatment should be adjusted according to the current symptoms and signs of the disease (axial, peripheral, enthesitis, extra-articular involvement, symptom, and clinical signs). Age, sex, comorbidities, and other medications are also important in the treatment plan. ${ }^{18}$ Owing to clinical heterogeneity of ax-SpA, individualized treatment was underlined. Clinicians should not rule out the total effect of extra-articular signs and comorbid situations. Since treatment is a long process, the preferences and expectations of the patient are of significance. Some patients do not accept the treatment because of adverse event risk. The treatment is individualistic since compliance and continuation is essential for treatment success. ${ }^{24}$ American College of Rheumatology (ACR) 2015 guideline discussed individualization of all treatment decisions. ${ }^{8}$

Ankylosing spondylitis affects young and productive patients and leaves severe social and psychological impacts. These factors impress patients' perception of the disease, outcomes, assessment and treatment process. Psychological comorbidity and social isolation problems decrease the treatment compliance and thereby treatment success. ${ }^{24}$

The first recommendation of the 2011 TLAR Recommendations was revised so as to comprise these parameters.

\section{Recommendation 2}

The monitoring of ax-SpA should consist of clinical signs, laboratory tests, patientreported outcome indices, and convenient imaging methods according to clinical signs, also considering the ASAS core set. The frequency of disease monitoring should be adjusted for each patient depending on symptoms, disease severity and treatment type. The disease activity should be assessed according to clinical signs and acute phase reactants. Ankylosing Spondylitis Disease Activity Score (ASDAS) is a preferred scale in ax-SpA, and should be used to determine treatment goals $(\mathrm{LoA}=9.21 \pm 1.47)$.

The great portion of this item is compatible with third recommendation of 2011 TLAR Recommendations. ${ }^{18}$ The term 'history of the patient' was altered.

In 2014 update of the Canadian Rheumatology Association/Spondyloarthritis Research Consortium of Canada, Treatment Recommendations for the Management of Spondyloarthritis Part I, the procedures to be taken were presented in 16 items (including quality of life, function, and work disability). ${ }^{10}$ Considering that patient-reported functional outcome indices, quality of life indicators, and work disability scales are used frequently, the term 'patient history' was changed as 'patient reported outcome indices'. It was also stated in this way in ASAS/EULAR 2016 Recommendations. ${ }^{4}$

The ASAS core set that includes function, pain, spinal mobility, and patient's global assessment is instructive in scrutinizing effects of diseasemodifying antirheumatic drugs (DMARDs), biologic DMARDs (bDMARDs), and physical functions. ${ }^{25}$

Bath Ankylosing Spondylitis Disease Activity Index (BASDAI) and ASDAS are two popular and important indices. Acute phase reactants have more prominent role in determining disease activity. 
ASDAS, a composite and more updated index, combines patient-based outcomes and C-reactive protein (CRP) or erythrocyte sedimentation rate. There are certain evidences that ASDAS more accurately reflects the inflammatory process and disease activity. While relationship between ASDAS and syndesmophyte formation is proven, the association with BASDAI is weaker. Therefore, ASDAS is a scale that may be preferred over BASDAI. ${ }^{5,9}$ These scales mainly focus on specific issues such as pain, disease activity, and physical function. However, they do not reflect the entire disease picture including impairment, limitations, restrictions, and social participation. Therefore, the ASAS Health Index, a composite index, was developed for the assessment of SpA patients at the basis of International Classification of Functioning, Disability and Health. ASAS Health Index will be used to test real life performance, and confirm performance of ax-SpA patients in clinical trials and daily practice. ${ }^{26}$ ASAS Health Index cut-off values for functioning categories were defined as follows: (1) normal functioning: $\leq 4$; (2) moderate functional impairment: 4-8 and (3) severe functional impairment: $\geq 8 .{ }^{27}$

In 2017 EULAR SpA imaging recommendations, MRI findings were also addressed. It was stated that extensive inflammatory signs in MRI (bone marrow edema) may be a predictor of good clinical response to anti-tumor necrosis factor (TNF) treatment. However, they also noted that MRI may help in making decision of anti-TNF therapy in association with clinical examination and CRP levels. In the same guideline, it was reported that 'MRI of the sacroiliac joints or the spine may be used for determination and follow-up of disease activity in ax-SpA, and this provides additional information beyond clinical and biochemical evaluation'. However, it was emphasized that there is no evidence on how frequently MRI should be performed to determine disease activity. ${ }^{12}$

\section{Recommendation 3}

Treatment goal should be 'remission' or 'inactive' disease. The treatment should be planned and conducted according to the treatment target. Low/minimal disease activity may be an alternative treatment target in some cases $(\mathrm{LoA}=9.39 \pm 1.74)$.
This recommendation explains the ideal treatment referred in the second item. The target is remission or inactive disease. The ideal remission is amelioration of symptoms and signs of the disease including the extra-articular findings, prevention of structural damage, and optimization of physical functions and quality of life.

The cut-off values of remission and inactive disease were determined for ASDAS and BASDAI in several studies. BASDAI scores above 4 indicate active disease. In some studies, BASDAI target was defined as $\leq 3$ in the $36^{\text {th }}$ week, and $50 \%$ reduction at sixth month; and minimal clinically important improvement was defined as 1.1 point reduction in BASDAI score. The limitation of ASDAS and BASDAI is that they do not involve extra-articular manifestations.

Assessment of SpondyloArthritis International Society partial remission criteria consist of patient's global assessment, spinal pain, physical function, Bath Ankylosing Spondylitis Functional Index and inflammation (BASDAI question 5, 6). The limitation of the criteria is that it does not contain objective inflammatory parameters and extra-articular features.

The ASDAS defines inactive disease as $<1.3$; moderate disease as 1.3-2; high disease activity as 2.1-3.5, and very high disease activity as $>3.5$ points. Minimal clinically important improvement refers to $\geq 1.1$; and major clinical improvement is $\geq 2.0$ points. ASDAS inactive disease value is the remission criteria and the target of treatment in ax-SpA. Clinical studies demonstrate that few patients attain this goal that harbors the risk of frequent treatment modifications and switching between biological agents. Moderate disease activity (1.3-2) may also be a target for treatment because low disease activity definition does not exist within the ASDAS. Since low disease activity may be misperceived that there is no disease activity, the term 'moderate disease activity' was preferred to reflect low-moderate disease activity. ${ }^{22}$

Expert panel discussed whether a 'window of opportunity' period as it is in RA existed for ax-SpA or not. In Outcome in AS International Study cohort, the radiographic course of the disease was searched scrutinizing patients every two years prospectively. As a result of 12 -years 
follow-up, it was depicted that structural damage was diverse and might not be predicted for majority of cases. Approximately 25\% of cases did not have radiographic progression while another $25 \%$ progressed rapidly. It was reported that progression and standstill periods occurred both in early and late phases, and progression was independent from disease and symptom duration. Radiographic progression was indicated to be faster in males and human leukocyte antigen B-27 positive individuals. This cohort also showed that patients on anti-TNF treatment had more rapid radiographic progression. However, this result was linked to the condition that patients receiving anti-TNF therapy are more active. It was seen that nonsteroidal anti-inflammatory drugs (NSAIDs) that are considered to have beneficial effects on radiographic progression were found not to have the exact effect over time. ${ }^{28}$ In further analysis of the same cohort, Ramiro et al. ${ }^{29}$ depicted that spinal radiographic progression is associated with disease activity and this association was more prominent in early disease stages. They emphasized the significance of early diagnosis, whereas no duration definition for window of opportunity was identified. Expert panel also concluded that it was not possible to define window of opportunity with current evidence.

\section{Recommendation $4 \mathbf{a}$}

The patient should be informed about his/her disease. This education should include relevant issues concerning his/her medical condition and treatment. The patient should be able to reach education in entire course of his/her disease. The patient should be encouraged for smoking cessation programs $(\mathrm{LoA}=9.75 \pm 2.09)$.

The fourth item of 2011 TLAR Recommendations was discussed under the title of third basic principle. The fifth recommendation of 2011 TLAR Recommendations: 'Nonpharmacological treatment approaches include mainly patient education, regular exercise programs (floor and water), balneotherapy and spa, physical therapy modalities and occupational therapy. 'Patient associations and help groups may be useful' was discussed in two items, '4a and $4 \mathrm{~b}$ ' (first refers to patient education and smoking cessation, and the latter refers to physical therapy and exercising). ${ }^{18}$
In some recommendations, patient education was emphasized to be an integral part of the treatment. ${ }^{4,14,17,18}$ Expert panel also discussed the importance of patient education and noted that sufficient weight is not given to this issue in daily practices despite having been emphasized in our prior national treatment recommendations. Nevertheless, there are certain studies showing that patient education contributed positively to coping with difficulties, pain, disability, and depression. ${ }^{14}$ The EULAR published a guideline to lead physicians, patients, health-care providers, policy-makers, and professional organizations in 2015. In this guideline, it was emphasized that patient education should be an interactive learning process to support patient management. Shared therapeutic decisions and adequate communication between the patient and health care provider are essential for education of patients with inflammatory arthritis. Patient education should be provided as an integral part of the standard care to enhance patients' participation and it should comprise the patient's medical condition and treatment (diagnosis, pharmacological and non-pharmacological treatment, physical and psychological condition) and should be accessible during disease duration., ${ }^{4,14}$

It is remarkable that smoking cessation programs are encouraged in last update of the ASAS/EULAR Recommendations. Cessation of smoking is important for human health and it occupies place in world health policy as well as ours. Both restrictive anomalies due to decreased spinal and thoracic cage mobility and intrinsic pulmonary pathologies including interstitial nodules and parenchymal problems may develop in the course of ax-SpA. ${ }^{30}$ Furthermore, in a study, it was shown that there was a relationship between smoking and disease activity, inflammation in MRI, and formation of syndesmophytes. ${ }^{31}$ Therefore, smoking cessation is of great significance for these patients.

\section{Recommendation $\mathbf{4 b}$}

Non-pharmacological treatment methods should be applied as soon as the diagnosis is established. An individualized exercise program adjusted according to clinical condition and expectations should be structured and applied. This program should be followed according to general principles and contraindications $(L o A=9.64 \pm 0.62)$. 
This item deals with exercise and other non-pharmacological treatment modalities. The Anatolian Group for the Assessment in Rheumatic Diseases which is a specific group of Turkish academicians (physical medicine and rehabilitation specialists and rheumatologists) who are experts in the rehabilitation of the patients with AS offered initiation of physical medicine and rehabilitation as early as possible after the diagnosis is established. Physiotherapy should be planned considering clinical condition, requirement, and expectations of the patient and followed regularly. Physiotherapy may be applied to all patients regardless of disease stage as outpatients or with hospitalization. It should be conducted parallel to general principles and contraindications. Life-long regular exercise is essential. ${ }^{15}$ Floor and water exercises, balneotherapy, spa, physical therapy modalities, and occupational therapy are among nonpharmacological treatment modalities. ${ }^{18}$ Although there is grade $1 \mathrm{~b}$ evidence and international consensus for significance of benefits of exercise in AS, detailed information to guide prescription of exercises is lacking. Therefore, evidence- based exercise consensus was published in 2016. This consensus recommends the type and dose of exercise that would be most beneficial. ${ }^{32}$ These recommendations also underline that exercise should be individualized. Individualization is based on objective parameters such as musculoskeletal and psychosocial factors, axial mobility, and chest expansion.

\section{Recommendation 5}

Patients complaining of pain and stiffness should use lowest effective doses of NSAIDs as first-line medication. Continuous use of NSAIDs should be preferred in patients responding well, and becoming symptomatic on discontinuation of NSAIDs. The risk and benefits should be taken into consideration in use of NSAIDs $(\operatorname{LoA}=9.07 \pm 1.82)$.

Several placebo-controlled trials have shown that NSAIDs are effective in SpA. Nevertheless, their therapeutic effect may differ from one patient to another. Good response to NSAIDs is a criterion for discrimination of inflammatory back pain. Effective pain control also provides functional improvement. While optimum response may be attained in some patients with medium doses, maximum tolerated dose may be required for others. The ASAS proposed an index for analysis and reporting of trials. This index deals with equivalent doses of NSAID. ${ }^{33}$ For the management of ax-SpA, NSAIDs were recommended at the "lowest effective dose" in the NICE guideline. ${ }^{6}$ Considering the chronic adverse effects of NSAIDs, we suggested "lowest effective dose". In 2011 TLAR Ankylosing Spondylitis National Recommendations, sixth recommendation was:"NSAIDs are the firstline agents to eliminate pain and stiffness. For patients harboring high risk for gastrointestinal adverse effects, NSAID and gastroprotective agent combinations or selective cyclooxygenase- 2 blockers may be preferred". Considering other adverse effects including cardiotoxic effects, the phrase "considering the risks and benefits" was preferred. NSAIDs have gastrointestinal, cardiovascular, and renal adverse effects, and they should be prescribed cautiously for patients with certain comorbidities. The potential side effects of NSAIDs should be taken into account particularly when used chronically. ${ }^{34}$ Therefore, considering the risks of long-term NSAID use, the question which patients should take NSAIDs continuously versus on demand is of pivotal role. Several studies have revealed that continuous use of NSAIDs in patients with higher CRP levels precludes structural damage of the spine. ${ }^{4}$ These studies support the hypothesis that NSAIDS may also have disease-modifying effects at long-term beyond anti-inflammatory effects. The question whether suppression of inflammation prevents structural damage or NSAIDs have direct inhibitory roles against ossification has not been clarified yet. ${ }^{35}$ Although NSAIDs are efficacious on the axial skeleton and systemic inflammation, there may be remaining systemic or axial inflammation even in patients whose symptoms are under control. ${ }^{36}$ Albeit high CRP levels or osteitis in MRI may demonstrate ongoing asymptomatic inflammation, current therapeutic approach bases on symptoms, thus drug or dose modifications are not recommended in good responders. ${ }^{37}$

\section{Recommendation 6}

In case of persistent pain, when
prior treatments are unsuccessful,
contraindicated, and/or intolerated,
paracetamol and opioid-like analgesics may
be added to the treatment (LoA=8.29 2.15$)$.


This item that was handled as sixth recommendation in ASAS/EULAR 2016 Recommendations was considered to be more convenient if discussed after pharmacological treatments by the expert panel. This item is compatible with seventh recommendation of 2011 TLAR Recommendations. ${ }^{18}$ However, the phrase "when NSAIDs are insufficient or contraindicated' was altered as 'when prior treatments are unsuccessful, contraindicated...' similar to sixth recommendation in ASAS/EULAR 2016 Recommendations. ${ }^{4}$

There are no prospective studies in the literature which assess use of paracetamol and simple analgesics in AS. A meta-analysis of 41 randomized studies conducted in 6019 patients revealed that opioids were superior to placebo in the management of chronic non-cancer pain (osteoarthritis, RA, central pain syndrome, phantom pain and fibromyalgia syndrome). Although their use in chronic pain is not thought to be related with misuse or abuse, it is not probable to make a definite conclusion because the studies have short durations, and their designs do not allow an appropriate evaluation of this aspect. $^{38}$

\section{Recommendation 7}

\section{Glucocorticoid injections may be performed for local inflammatory conditions of the musculoskeletal system. Long-term systemic glucocorticoid use is not recommended for patients with axial involvement ( $L A A=8.64 \pm 2.24)$.}

The recommendation regarding local injections is similar to 2011 TLAR Recommendations and it was reemphasized that local glucocorticoid (GC) injection may be an option in treatment of arthritis and enthesitis. ${ }^{18}$ The formulation regarding systemic use of GCs has changed slightly. It was shown that shortterm high dose GC use is beneficial to improve clinical symptom and signs. ${ }^{39,40}$ Therefore, the phrase 'there is no evidence in use of systemic GCs in ax-SpA' was altered as 'long-term systemic GCs are not recommended', which is compatible with 2016 update of the ASAS/ EULAR Management Recommendations for ax-SpA. ${ }^{4}$

\section{Recommendation 8}

Conventional synthetic DMARDs are not recommended for patients with pure axial involvement normally. Sulfasalazine (SSZ) may be considered for patients with peripheral arthritis $(\mathrm{LoA}=\mathbf{8 . 7 1} \pm \mathbf{1 . 8 3})$.

This item was handled in 2011 TLAR Recommendations as follows; 'There is no evidence regarding the effect of disease-modifying agents (including SSZ, methotrexate) on axial disease, but SSZ may be used in peripheral arthritis'. ${ }^{18}$

In a meta-analysis, it was revealed that methotrexate was not efficacious in AS. ${ }^{41}$ Also, there is no evidence regarding efficacy of leflunomide in AS. ${ }^{42}$ There is consensus on the opinion that patients with pure axial disease should not be treated with conventional synthetic DMARDs and there is evidence that SSZ, methotrexate, and leflunomide are not efficacious for axial symptoms, therefore the term "normally" was added to 2016 ASAS/ EULAR and 'exceptional situations' where other treatment options may not be considered due to toxicity, contraindications, or costs were emphasized. ${ }^{4} 2015$ ACR/Spondylitis Association of America/Spondyloarthritis Research and Treatment Network panel also recommended use of conventional synthetic DMARDs in active patients despite NSAID use in whom TNFinhibitors (TNFis) are contraindicated. SSZ is known to be inefficacious in ax-SpA. The role of low-cost treatment agents such as SSZ in early stage is yet of unknown significance. In the Effect of Etanercept versus Sulfasalazine in Early Axial Spondyloarthritis on Active Inflammatory Lesions as Detected by Whole-body MRI (ESTHER) trial, the ASAS 20 and ASAS 40 responses were $85 \%$ and $70 \%$ in etanercept (ETA) group, and $42 \%$ and $31 \%$ in SSZ group. The rates attained with SSZ were lower when compared to ETA, but relatively good. The improvement in MRI scores at the end of the first year was $35.2 \%$ in SSZ, and 69.2\% in ETA groups. Therefore, further studies on efficacy of SSZ in early stage are required. ${ }^{11,32,43}$

\section{Recommendation 9}

Use of bDMARDs (the current practice is to start with a TNFi) should be considered for the patients with high disease activity despite standard treatments (LoA=9.75 \pm 0.58). 
The part with 'anti-TNFs' in $10^{\text {th }}$ item of 2011 TLAR Recommendations was changed as 'bDMARD' as in accordance with 2016 ASAS/ EULAR Recommendations. In 2011, the term 'anti-TNF' was preferred as the term 'bDMARD' or another synonym was not available. Six TNFi agents approved for treatment of ax-SpA exist in our country; infliximab, ETA, adalimumab, golimumab, certolizumab, and CT-P13, an infliximab biosimilar., ${ }^{48,42}$ In meta-analysis on efficacy and safety of biologic agents in AS, it was found that TNFi agents were not superior over another, and their safety profiles were similar (injection site reaction, infection, severe infection, drug discontinuation due to adverse effects). ${ }^{4,45}$ Novel studies report that TNFi agents provide similar improvement in terms of disease activity and functionality in early non-radiographic ax-SpA patients. ${ }^{46,47}$ Randomized controlled trials of CT-P13, an infliximab biosimilar indicate that it has similar efficacy and safety with the innovator infliximab. $^{48,49}$ Predictors for good response to TNFi are short disease duration, $\leq 40$ years, absence of enthesitis, human leukocyte antigen B-27 positivity, good functional status, and high CRP levels. ${ }^{50}$

Tumor necrosis factor-alpha blocking agents may be used efficiently for the treatment of uveitis, dactylitis, enthesitis, psoriasis, and inflammatory bowel diseases. There are evidences that monoclonal TNFi are superior to ETA in inflammatory bowel disease and uveitis. ${ }^{11,19}$

In determination of active disease in patients that are candidates for biological treatment, two occasions are present; ASDAS $>2.1$ or BASDAI $>4$. As discussed in the third recommendation, ASDAS reflects the inflammatory condition better than BASDAI and its cut-off values are preferable. Physician's opinion is also important in determination of the disease activity. The decision of active disease is based on clinical, laboratory (CRP), and imaging (MRI) findings., ${ }^{4,5}$ Non-responsiveness to conventional therapy is defined as active disease despite using at least two different NSAIDs at maximum anti-inflammatory dose and duration (at least two weeks for each). Intolerance or adverse effects are also within this scope. Patients with peripheral arthritis are accepted as non-responsive to conventional synthetic DMARDs after failure with three months of full dose SSZ use and local GC injections. ${ }^{9}$ These patients are candidates for bDMARD therapy.

In 2016, another bDMARD acting on a different pathway has emerged: interleukin (IL)-17 inhibition. Up to date, data on IL-17 inhibition in radiographic AS are present; however, data on non-radiographic ax-SpA patients is lacking. Secukinumab has recently been approved in our country, so our experience is not enough.

Experiences with TNFi regarding efficacy and safety in ax-SpA and certain indications are abundant. Therefore, beginning with a TNFi is recommended. ${ }^{47,51-54}$

\section{Recommendation 10}

\section{In case of failed TNFi treatment, switching to another TNFi or an IL-17i should be thought $(\mathrm{Lo} A=9.61 \pm 0.68)$.}

The response to biologics should be assessed after three months of continuous use. The response criteria are defined as a decrease of $\geq 1.1$ in ASDAS and $\geq 50 \%$ or two units (over a 0-10 scale) in BASDAI. ${ }^{9}$

Among TNFi users, switching to another TNFi due to primary non-responsiveness was reported to be $14 \%-68 \%$, secondary nonresponsiveness $13-61 \%$, and adverse effects or intolerance $13-57 \% .{ }^{50,55} \mathrm{TNFi}$ switch is more common in females, elderly patients, those with more symptoms, complete ankylosis, enthesitis, and higher erythrocyte sedimentation rate values. Switching the TNFi was found to be beneficial without increasing adverse effects in ax-SpA patients non-responding to first and also second TNFi. TNFi switch is recommended in international guidelines in case of non-response. , $^{41,50}$

Tumor necrosis factor inhibitor agents and secukinumab are accepted to be equivalent in case of switching in ASAS/EULAR Recommendations. However, the patients to be preferred for IL-17i are ones with higher risk for tuberculosis, psoriasis, and severe peripheral arthritis. IL-17i is not preferred in inflammatory bowel disease. ${ }^{51}$

\section{Recommendation 11}

\section{In patients with persistent remission, tapering bDMARDs may be thought (LoA=9.32 \pm 1.31$)$.}


This recommendation was absent in prior national or international guidelines. This principle that is previously included in "2016 ASAS/EULAR Update of Management Recommendations for Axial Spondyloarthritis" and "Portuguese Recommendations for the Use of Biological Therapies in Patients with Axial Spondyloarthritis-2016 Update" was incorporated to our recommendations for the first time., ${ }^{4,9}$ Complete withdrawal of TNFi results in flares. On the other hand, high treatment costs and potential adverse effects have induced opinion of tapering biologics in patients with sustained remission. However, the exact duration for "sustained remission" is unclear. ASAS/EULAR defines it as six months or above, but the expert panel stated that it should be at least one year. The methods to follow for tapering are reducing the dose or extending the interval between doses. This should be based on a shared decision between patient and the physician. ${ }^{4,9}$

\section{Recommendation $12 \mathrm{a}$}

In the presence of refractory pain or functional impairment and radiographic structural damage; total hip arthroplasty should be thought independent of age $(\mathrm{Lo} A=9.46 \pm 0.88)$.

\section{Recommendation $\mathbf{1 2 b}$}

Spinal surgical interventions such as corrective osteotomy or stabilization in specialized centers may provide benefit in selected cases with severe, disabling spinal deformity ( $L o A=8.61 \pm 1.39)$.

In TLAR 2011 Recommendations, this item was handled as the $11^{\text {th }}$ item; "Total hip arthroplasty should be considered in patients with refractory pain or disability and radiographic evidence of structural damage, independent of age. In selected cases, spinal surgical interventions such as corrective osteotomy or stabilization may provide benefit". The first part of the prior $11^{\text {th }}$ recommendation was taken to recommendation $12 \mathrm{a}$ exactly the same. The second sentence of the prior $11^{\text {th }}$ item was discussed as $12 \mathrm{~b}$ and in accordance with 2016 update of the ASAS/ EULAR Management Recommendations for Axial SpondyloArthritis, and the terms "severe disabling spinal deformity" and "specialized centers" were added. The goal is to emphasize the significance of discussing benefits and risks of spinal corrective surgery.

The level of evidence for total hip arthroplasty is " $\mathrm{C}$ ". Severe damage necessitating joint replacement may occur in patients with SpA. Particularly in patients whom inflammation cannot be controlled, arthroplasty requirement may evolve much earlier than it occurs in the course of degenerative diseases. Therefore, for arthroplasty, the term 'independent of age' is of importance. ${ }^{4,6}$

Arthroplasty enhances mobility and quality of life. Nevertheless, comorbid situations should be taken into account when decision for surgery is made. The operation should be performed by an orthopedist in experienced centers about joint replacement in AS patients. ${ }^{8}$

The success of arthroplasty is influenced by several factors including the medication patient uses. Thus, ACR published a guideline to help clinicians for elective surgeries. In this guideline, discontinuation of all bDMARDs prior to surgery, and planning surgery at the end of dosing period of each drug were recommended on conditional basis. Following the wound healing (approximately 14 days) and after sutures are removed, if the wound site is clean, treatment may be readministered on conditional basis. ${ }^{16}$

Progressive spinal deformity that interferes with horizontal view, walking, and interaction may develop in ax-SpA patients. Corrective osteotomies may be considered in these patients. However, these procedures should be performed in specialized centers by spinal surgeons with spinal surgery training after a meticulous evaluation. ${ }^{4,6}$ The NICE guideline notes that this complex surgery should be performed on patients with severely impaired quality of life and progressive deformity despite non-surgical treatment modalities. ${ }^{6}$ ACR is conditionally against elective spinal surgery. ${ }^{8}$ French Society reported that this indication is rare. ${ }^{7}$ Therefore, we preferred the term 'selected cases' in recommendation $12 \mathrm{~b}$.

\section{Recommendation 13a}

Risk factors regarding cardiovascular comorbidities should be overviewed in all ax-SpA patients $(\mathrm{LoA}=9.07 \pm 1.56)$. 
Neither TLAR 2011 nor ASAS/EULAR Recommendations refers to cardiovascular risk in a separate item. Comorbid situations were mentioned in basic principle 2. The most important complications in the long-term belong to cardiovascular system. ${ }^{6}$ Therefore, the expert panel decided to add this recommendation to increase awareness regarding cardiovascular risk.

The risk for cardiovascular diseases (CVDs) is especially higher in patients whom disease activity cannot be controlled adequately. This may be due to either direct vascular effect or decreased cardiovascular fitness. Long-term use of NSAIDs is considered to increase CVD risk whereas their antiinflammatory effects may also reduce this risk. ${ }^{6}$

The most common risk factors reported in ASAS-COMOrbidities in SPondyloArthritis study are hypertension, smoking and hypercholesterolemia. Other factors are family history and diabetes. All risk factors should be scrutinized optimally. ${ }^{56}$

In EULAR Recommendations for CVD risk management in patients with RA and other forms of inflammatory arthritides, it was reported that clinicians should be aware that the risk is higher than general population and should check for the risk factors. Tight control of the disease activity would decrease CVD risk. Management of CVD risk should be performed according to national guidelines in a multidisciplinary approach. The same recommendation also contains the general principle that NSAIDs and GCs should be used in accordance with ASAS/ EULAR guidelines. ${ }^{13}$

\section{Recommendation $13 \mathrm{~b}$}

Patients with ax-SpA should be informed about increased fracture risk. They should be assessed in terms of osteoporosis (LoA=9.50 \pm 0.79).

Neither 2011 TLAR Recommendations nor 2016 update of the ASAS/EULAR Recommendations have an item about fracture risk or osteoporosis. In ASAS-COMOrbidities in SPondyloArthritis study, osteoporosis was found to be the most common comorbidity, but prevalence of vertebra and proximal humerus fractures were low. The NICE and ACR guidelines mention osteoporosis and fracture risk. Informing patients in terms of fracture risk and assessment of fall risk was recommended. 6,8,56

The NICE guideline recommends biennially osteoporosis assessment for Ax-SpA patients. Dual energy X-ray absorptiometry scores were underlined to be high due to syndesmophytes and ligament calcification, and hip scores were recommended in this scope. Falling may cause fractures or neurological damage. The risk for falls is higher among patients with osteoporosis, intensive spinal fusion, postural instability, and other neurological or musculoskeletal diseases that may affect balance. 6,8 ACR recommends that screening program for osteoporosis should be performed considering the age, sex, physical activity state, disease severity and duration, and other risk factors. ${ }^{8}$

\section{Recommendation 13c}

The spinal pain evolving abruptly and not considered to be related to inflammation should be investigated, appropriate evaluation including imaging should be performed. In case of acute vertebral fracture, consultation to a spinal surgeon and examination should assuredly be sought $(\mathrm{LoA}=9.39 \pm 0.99)$.

This item was taken from TLAR 2011 Recommendations, and 'evaluation including imaging' was added from ASAS/EULAR Recommendations. Grade of evidence is D. Fusioned spinal segment fractures harbor risks of instability and neurological complications. ${ }^{4,7}$

Patients with high and persistent disease activity, long-term disease, occiput to wall distance $>0$, or persistent pain after minimal trauma should be regarded as AS-specific highrisk groups. Radiographic evaluation is necessary for the patients with persistent pain for early diagnosis and treatment. As mentioned earlier, in case of acute vertebral fracture, the spinal surgery consultation should be needed. Also, infections (vertebral, discogenic, or epidural) and metastatic and primary tumors should be kept in mind in differential diagnosis. Appropriate laboratory and imaging approaches should also be performed. ${ }^{6,7,18}$ The basic principles and recommendations are summarized in Table 3. Algorithm for patient assessment 
Table 3. TLAR Expert Opinions for Axial Spondyloarthritis Treatment

$\begin{array}{cc}\text { Level of } & \text { Strength of } \\ \text { agreement LoA } & \text { recommendation }\end{array}$

\section{Basic Principles}

1. Ax-SpA is a potentially severe disease. A multidisciplinary approach in coordination of a

$9.29 \pm 2.22$ rheumatologist or physical medicine and rehabilitation specialist is required for the management of musculoskeletal and extraarticular manifestations

2. The main aims of the treatment of patients with ax-SpA are to control the inflammation and symptoms, to prevent structural damage, to minimize comorbidities, to avoid adverse effects of the treatment, normalization and preservation of functionality and social participation, and maximization of the health-related quality of life.

3. Non-pharmacological and pharmacological treatment methods should be combined in the ideal treatment of patients with ax-SpA.

4. The treatment of ax-SpA should rely on the shared decision between patient and physician, and should aim the best treatment and care.

5. Ax-SpA has high personal, medical, and societal costs. All of them should be kept in mind in the management of the disease.

\section{Recommendations}

1. The treatment of ax-SpA patients should be individualized considering current symptom and signs of the disease (axial, peripheral, extraarticular), comorbid situations, psychosocial factors, request and expectations of patients.

2. The monitoring of ax-SpA should consist of clinical signs, laboratory tests, patient-reported outcome indices, and convenient imaging methods according to clinical signs, also considering the ASAS core set*. The frequency of disease monitoring should be adjusted for each patient depending on symptoms, disease severity, and treatment type*. The disease activity should be assessed according to clinical signs and acute phase reactants**. ASDAS is a preferred scale in ax-SpA, and should be used to determine treatment goals**.

3. Treatment goal should be remission or inactive disease. The treatment should be planned and conducted according to treatment target. Low/minimal disease activity may be an alternative treatment target in some cases.

4a. Patient should be informed about his/her disease*. This education should include relevant issues concerning his/her medical condition and treatment**. Patient should be able to reach education in entire course of his/her disease**. Patient should be encouraged for smoking cessation programs**.

4b. Non-pharmacological treatment methods should be applied as soon as the diagnosis is established*. An individualized exercise program adjusted according to clinical condition and expectations should be structured and applied**. This program should be followed according to general principles and contraindications ${ }^{* * *}$.

5. Patients complaining of pain and stiffness should use lowest effective doses of NSAIDs as firstline medication. Continuous use of NSAIDs should be preferred in patients responding well, and becoming symptomatic on discontinuation of NSAIDs. The risk and benefits should be taken into consideration in use of NSAIDs.

6. In case of persistent pain, when prior treatments are unsuccessful, contraindicated, and/or intolerated, paracetamol and opioid-like analgesics may be added to the treatment.

7. Glucocorticoid injections may be performed for local inflammatory conditions of the musculoskeletal system*. Long-term systemic glucocorticoid use is not recommended for patients with axial involvement**.

8. Conventional synthetic DMARDs are not recommended for patients with pure axial involvement normally. Sulfasalazine may be considered for patients with peripheral arthritis.

9. Use of bDMARDs (the current practice is to start with a TNFi ) should be considered for the patients with high disease activity despite standard treatments.

10. In case of failed TNFi treatment, switching to another TNFi* or a IL17i** should be thought.

11. In patients with persistent remission, tapering bDMARDs may be thought.

12a. In the presence of refractory pain or functional impairment and radiographic structural damage; total hip arthroplasty should be thought independent of age.

12b. Spinal surgical interventions such as corrective osteotomy or stabilization in specialized centers may provide benefit in selected cases with severe, disabling spinal deformity.

13a. Risk factors regarding cardiovascular comorbidities should be overviewed in all ax-SpA patients.

13b. Patients with ax-SpA should be informed about increased fracture risk. They should be assessed in terms of osteoporosis.

13c. The spinal pain evolving abruptly and not considered to be related to inflammation should be investigated, appropriate evaluation including imaging should be performed. In case of acute vertebral fracture, consultation of a spinal surgeon and examination should assuredly be sought.

$9.89 \pm 0.31$

$9.25 \pm 2.35$

$9.36 \pm 1.74$

$9.18 \pm 1.76$

$9.36 \pm 1.74$ 
PATIENT ASSESSMENT AND THERAPEUTIC ALGORITHM

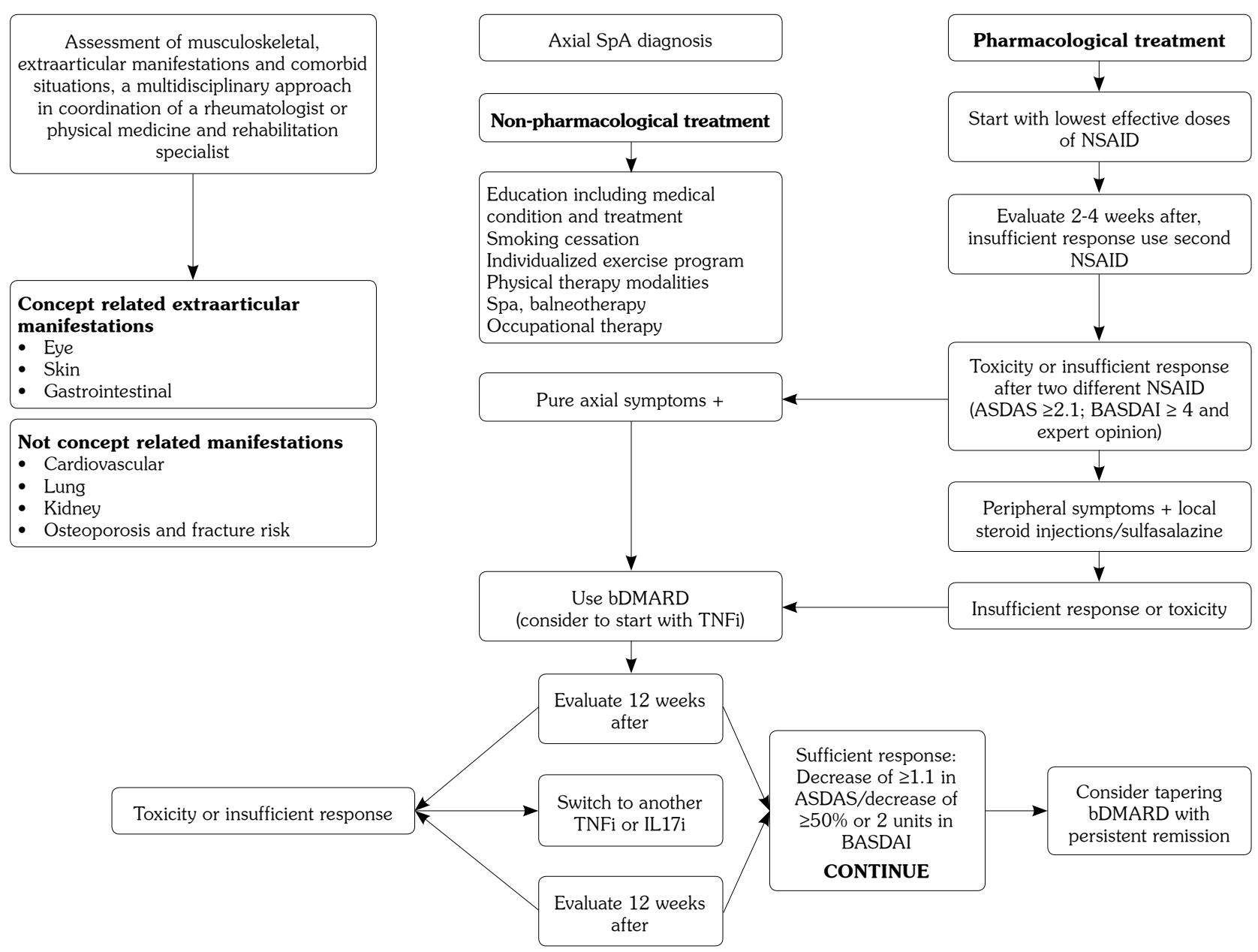

Figure 1. Algorithm for patient assessment and management of axial spondyloarthritis. SpA: Spondyloarthritis; NSAID: Nonsteroidal antiinflammatory drugs; ASDAS: Ankylosing Spondylitis Disease Activity Score; BASDAI: Bath Ankylosing Spondylitis Disease Activity index; bDAMRD: Biologic disease-modifying antirheumatic drugs; TNFi: Tumor Necrosis Factor alpha inhibitor; IL-17i: Interleukin-17 inhibitor.

and management of axial spondyloarthritis summarized in Figure 1.

In conclusion, these opinions were based on current guidelines and the literature on the topic. The recommendations in the present study have sufficient agreement levels. It is hoped that these recommendations will facilitate ax-SpA management in daily clinical practice among our colleagues.

\section{Declaration of conflicting interests}

The authors declared no conflicts of interest with respect to the authorship and/or publication of this article.

\section{Funding}

The authors received no financial support for the research and/or authorship of this article.

\section{REFERENCES}

1. Sieper J, Poddubnyy D. Axial spondyloarthritis. Lancet 2017;390:73-84.

2. Yu DT. Diagnosis and differential diagnosis of ankylosing spondylitis in adults. In: Sieper J, section editor. Avaliable from: https://www.uptodate.com/contents/search

3. van der Heijde D, Aletaha D, Carmona L, Edwards CJ, Kvien TK, Kouloumas M, et al. 2014 Update of the EULAR standardised operating procedures for EULAR-endorsed recommendations. Ann Rheum Dis 2015;74:8-13. 
4. van der Heijde D, Ramiro S, Landewé R, Baraliakos X, Van den Bosch F, Sepriano A, et al. 2016 update of the ASAS-EULAR management recommendations for axial spondyloarthritis. Ann Rheum Dis 2017;76:978-91.

5. Smolen JS, Schöls M, Braun J, Dougados M, FitzGerald O, Gladman DD, et al. Treating axial spondyloarthritis and peripheral spondyloarthritis, especially psoriatic arthritis, to target: 2017 update of recommendations by an international task force. Ann Rheum Dis 2018;77:3-17.

6. National Institute for Health and Care Excellence: Clinical Guidelines. Spondyloarthritis in Over 16s: Diagnosis and Management. London: NICE Guideline No 65; 2017.

7. Wendling D, Lukas C, Paccou J, Claudepierre P, Carton L, Combe $\mathrm{B}$, et al. Recommendations of the French Society for Rheumatology (SFR) on the everyday management of patients with spondyloarthritis. Joint Bone Spine 2014;81:6-14.

8. Ward MM, Deodhar A, Akl EA, Lui A, Ermann J, Gensler LS, et al. American College of Rheumatology/Spondylitis Association of America/Spondyloarthritis Research and Treatment Network 2015 Recommendations for the Treatment of Ankylosing Spondylitis and Nonradiographic Axial Spondyloarthritis. Arthritis Rheumatol 2016;68:282-98.

9. Machado P, Cerqueira M, Ávila-Ribeiro P, Aguiar R, Bernardo A, Sepriano A, et al. Portuguese recommendations for the use of biological therapies in patients with axial spondyloarthritis - 2016 update. Acta Reumatol Port 2017;42:209-18.

10. Rohekar S, Chan J, Tse SM, Haroon N, Chandran V, Bessette L, et al. 2014 Update of the Canadian Rheumatology Association/spondyloarthritis research consortium of Canada treatment recommendations for the management of spondyloarthritis. Part I: principles of the management of spondyloarthritis in Canada. J Rheumatol 2015;42:654-64.

11. Rohekar S, Chan J, Tse SM, Haroon N, ChandranV, Bessette L, etal. 2014Update of the Canadian Rheumatology Association/Spondyloarthritis Research Consortium of Canada Treatment Recommendations for the Management of Spondyloarthritis. Part II: Specific Management Recommendations. J Rheumatol 2015;42:665-81.

12. Mandl P, Navarro-Compán V, Terslev L, Aegerter $\mathrm{P}$, van der Heijde D, D'Agostino MA, et al. EULAR recommendations for the use of imaging in the diagnosis and management of spondyloarthritis in clinical practice. Ann Rheum Dis 2015;74:1327-39.

13. Agca R, Heslinga SC, Rollefstad S, Heslinga M, McInnes IB, Peters MJ, et al. EULAR recommendations for cardiovascular disease risk management in patients with rheumatoid arthritis and other forms of inflammatory joint disorders: 2015/2016 update. Ann Rheum Dis 2017;76:17-28.

14. Zangi HA, Ndosi M, Adams J, Andersen L, Bode C, Boström C, et al. EULAR recommendations for patient education for people with inflammatory arthritis. Ann Rheum Dis 2015;74:954-62.

15. Ozgocmen S, Akgul O, Altay Z, Altindag O, Baysal O, Calis $\mathrm{M}$, et al. Expert opinion and key recommendations for the physical therapy and rehabilitation of patients with ankylosing spondylitis. Int $J$ Rheum Dis 2012;15:229-38.

16. Goodman SM, Springer B, Guyatt G, Abdel MP, Dasa V, George M, et al. 2017 American College of Rheumatology/American Association of Hip and Knee Surgeons Guideline for the Perioperative Management of Antirheumatic Medication in Patients With Rheumatic Diseases Undergoing Elective Total Hip or Total Knee Arthroplasty. J Arthroplasty 2017;32:2628-38.

17. Combe B, Landewe R, Daien CI, Hua C, Aletaha D, Álvaro-Gracia JM, et al. 2016 update of the EULAR recommendations for the management of early arthritis. Ann Rheum Dis 2017;76:948-59.

18. Bodur H, Sivas F, Yılmaz Ö, Özgöçmen S, Günaydın. $\mathrm{R}$, Kaya $\mathrm{T}$, et al. Turkish League against Rheumatism. National Recommendations for the management of ankylosing spondylitis. Turk $\mathrm{J}$ Rheumatol 2011;26:173-86.

19. Rosenbaum J, Chandran V. Management of comorbidities in ankylosing spondylitis. Am J Med Sci 2012;343:364-6.

20. van der Horst-Bruinsma I, de Vries M, van den Bosch F. Spondyloarthritis: Treatment. In: Bijlsma JWJ, Hachulla E, editors EULAR Textbook on Rheumatic Disease. 2nd ed. London: BMJ; 2015. p. 319-43.

21. Bodur $\mathrm{H}$. The Fight Against Rheumatism is in its $70^{\text {th }}$ Year. Arch Rheumatol 2017;32:1-2.

22. Danve A, Deodhar A. Treat to Target in Axial Spondyloarthritis: What Are the Issues? Curr Rheumatol Rep 2017;19:22.

23. Reimold AM, Chandran V. Nonpharmacologic therapies in spondyloarthritis. Best Pract Res Clin Rheumatol 2014;28:779-92.

24. Bergman M, Lundholm A. Managing morbidity and treatment-related toxicity in patients with ankylosing spondylitis. Rheumatology 2018;57:419-28.

25. Sieper J, Rudwaleit M, Baraliakos X, Brandt J, Braun J, Burgos-Vargas R, et al. The Assessment of SpondyloArthritis international Society (ASAS) handbook: a guide to assess spondyloarthritis. Ann Rheum Dis 2009;68 Suppl 2:ii1-44.

26. Landewé R, van Tubergen A. Clinical Tools to Assess and Monitor Spondyloarthritis. Curr Rheumatol Rep 2015;17:47.

27. Di Carlo M, Lato V, Di Matteo A, Carotti M, Salaffi F. Defining functioning categories in axial Spondyloarthritis: the role of the ASAS Health Index. Rheumatol Int 2017;37:713-8.

28. Ramiro S, Stolwijk C, van Tubergen A, van der Heijde $\mathrm{D}$, Dougados M, van den Bosch F, et al. Evolution of radiographic damage in ankylosing spondylitis: a 12 year prospective follow-up of the OASIS study. Ann Rheum Dis 2015;74:52-9.

29. Ramiro S, van der Heijde $\mathrm{D}$, van Tubergen $\mathrm{A}$, Stolwijk C, Dougados M, van den Bosch F, et al. Higher disease activity leads to more structural damage in the spine in ankylosing spondylitis: 12-year longitudinal data from the OASIS cohort. Ann Rheum Dis 2014;73:1455-61. 
30. Berdal G, Halvorsen S, van der Heijde D, Mowe M, Dagfinrud H. Restrictive pulmonary function is more prevalent in patients with ankylosing spondylitis than in matched population controls and is associated with impaired spinal mobility: a comparative study. Arthritis Res Ther 2012;14:19.

31. Poddubnyy D, Haibel H, Listing J, Märker-Hermann $\mathrm{E}$, Zeidler $\mathrm{H}$, Braun $\mathrm{J}$, et al. Baseline radiographic damage, elevated acute-phase reactant levels, and cigarette smoking status predict spinal radiographic progression in early axial spondylarthritis. Arthritis Rheum 2012;64:1388-98.

32. Millner JR, Barron JS, Beinke KM, Butterworth RH, Chasle BE, Dutton LJ, et al. Exercise for ankylosing spondylitis: An evidence-based consensus statement. Semin Arthritis Rheum 2016;45:411-27.

33. Bodur H. Treatment of Early Axial Spondyloarthritis. Turkiye Klinikleri J PM\&R-Special Topics 2014;7:9-15.

34. Solomon DH. "Nonselective NSAID: Overview of aderverse effects" In: Furst DE, section editor. Avaliable from: https://www.uptodate.com/contents/search

35. Poddubnyy D, Rudwaleit M, Haibel H, Listing J, MärkerHermann E, Zeidler $\mathrm{H}$, et al. Effect of non-steroidal antiinflammatory drugs on radiographic spinal progression in patients with axial spondyloarthritis: results from the German Spondyloarthritis Inception Cohort. Ann Rheum Dis 2012;71:1616-22.

36. Baraliakos X, Kiltz U, Peters S, Appel H, Dybowski F, Igelmann $\mathrm{M}$, et al. Efficiency of treatment with nonsteroidal anti-inflammatory drugs according to current recommendations in patients with radiographic and non-radiographic axial spondyloarthritis. Rheumatology (Oxford) 2017;56:95-102.

37. Poddubnyy D, van der Heijde D. Therapeutic controversies in spondyloarthritis: nonsteroidal anti-inflammatory drugs. Rheum Dis Clin North Am 2012;38:601-11.

38. Furlan AD, Sandoval JA, Mailis-Gagnon A, Tunks E. Opioids for chronic noncancer pain: a metaanalysis of effectiveness and side effects. CMAJ 2006;174:1589-94.

39. Haibel H, Fendler C, Listing J, Callhoff J, Braun $\mathrm{J}$, Sieper J. Efficacy of oral prednisolone in active ankylosing spondylitis: results of a double-blind, randomised, placebo-controlled short-term trial. Ann Rheum Dis 2014;73:243-6.

40. Bandinelli F, Scazzariello F, Pimenta da Fonseca E, Barreto Santiago M, Marcassa C, Nacci F, et al. Low-dose modified-release prednisone in axial spondyloarthritis: 3-month efficacy and tolerability. Drug Des Devel Ther 2016;10:3717-3724.

41. Chen J, Veras MM, Liu C, Lin J. Methotrexate for ankylosing spondylitis. Cochrane Database Syst Rev 2013;2:CD004524.

42. Kılıç G, Özgöçmen S. Pharmacological management of ankylosing spondylitis. Turkiye Klinikleri J PM\&RSpecial Topics 2014;7:35-43.

43. Song $\mathrm{IH}$, Hermann K, Haibel H, Althoff CE, Listing $\mathrm{J}$, Burmester $\mathrm{G}$, et al. Effects of etanercept versus sulfasalazine in early axial spondyloarthritis on active inflammatory lesions as detected by whole-body MRI (ESTHER): a 48-week randomised controlled trial. Ann Rheum Dis 2011;70:590-6.

44. Chen C, Zhang X, Xiao L, Zhang X, Ma X. Comparative Effectiveness of Biologic Therapy Regimens for Ankylosing Spondylitis: A Systematic Review and a Network Meta-Analysis. Medicine (Baltimore) 2016;95:3060.

45. Ma Z, Liu X, Xu X, Jiang J, Zhou J, Wang J, et al. Safety of tumor necrosis factor-alpha inhibitors for treatment of ankylosing spondylitis: A meta-analysis. Medicine (Baltimore) 2017;96:7145.

46. Callhoff J, Sieper J, Weiß A, Zink A, Listing J. Efficacy of TNF- $\alpha$ blockers in patients with ankylosing spondylitis and non-radiographic axial spondyloarthritis: a metaanalysis. Ann Rheum Dis 2015;74:1241-8.

47. Sieper J, Poddubnyy D. New evidence on the management of spondyloarthritis. Nat Rev Rheumatol 2016;12:282-95.

48. Yu DT, Assessment and Treatment for Ankylosing Spondylitis in Adults- Biosimiliar for Infliximab. In: Sieper J, section editor. Avaliable from: https://www. uptodate.com/contents/search.

49. Glintborg B, Sørensen IJ, Loft AG, Lindegaard H, Linauskas A, Hendricks $\mathrm{O}$, et al. A nationwide nonmedical switch from originator infliximab to biosimilar CT-P13 in 802 patients with inflammatory arthritis: 1-year clinical outcomes from the DANBIO registry. Ann Rheum Dis 2017;76:1426-31.

50. Deodhar A, Yu D. Switching tumor necrosis factor inhibitors in the treatment of axial spondyloarthritis. Semin Arthritis Rheum 2017;47:343-50.

51. Cheung PP. Anti-IL17A in Axial SpondyloarthritisWhere Are We At? Front Med (Lausanne) 2017;4:1.

52. Sieper J, Deodhar A, Marzo-Ortega H, Aelion JA, Blanco R, Jui-Cheng $T$, et al. Secukinumab efficacy in anti-TNF-naive and anti-TNF-experienced subjects with active ankylosing spondylitis: results from the MEASURE 2 Study. Ann Rheum Dis 2017;76:571-92.

53. Baeten D, Sieper J, Braun J, Baraliakos X, Dougados M, Emery P, et al. Secukinumab, an Interleukin-17A Inhibitor, in Ankylosing Spondylitis. N Engl J Med 2015;373:2534-48.

54. Braun J, Baraliakos X, Deodhar A, Baeten D, Sieper J, Emery $\mathrm{P}$, et al. Effect of secukinumab on clinical and radiographic outcomes in ankylosing spondylitis: 2-year results from the randomised phase III MEASURE 1 study. Ann Rheum Dis 2017;76:1070-7.

55. Cantini F, Niccoli L, Nannini C, Cassarà E, Kaloudi $\mathrm{O}$, Giulio Favalli E, et al. Second-line biologic therapy optimization in rheumatoid arthritis, psoriatic arthritis, and ankylosing spondylitis. Semin Arthritis Rheum 2017;47:183-92.

56. Moltó A, Etcheto A, van der Heijde D, Landewé R, van den Bosch F, Bautista Molano W, et al. Prevalence of comorbidities and evaluation of their screening in spondyloarthritis: results of the international crosssectional ASAS-COMOSPA study. Ann Rheum Dis 2016;75:1016-23. 